\title{
On the Sensitivity of a Laser Heterodyne Polarimeter for Vacuum Birefringence Detection
}

\author{
Gabriel Alberts $^{1}$, Hal Hollis ${ }^{1}$, Harrison LaBollita ${ }^{2}$ \\ ${ }^{1}$ College of Liberal Arts and Sciences, University of Florida \\ ${ }^{2}$ Department of Mathematics and Physics, Piedmont College
}

Faculty Mentor: Guido Mueller, Department of Physics

\begin{abstract}
Detecting vacuum magnetic birefringence (VMB) requires an immensely accurate, precisely calibrated experiment. We are working on a new design to (1) detect the birefringence (BF) of individual optical components without using a cavity, and (2) measure VMB in reflection off a cavity. Our design uses two overlapping, orthogonally polarized laser beams to measure the relative phase difference between a reference path and one with rotating polarizations, which may experience oscillating phase shifts in vacuum in the presence of a magnetic field. To test the design, we developed a small-scale laser heterodyne polarimeter (LHP) without cavities that can analyze different birefringent sources with the same principle. Our early results from testing mirrors show spatial variations in BF due to imperfect mirror coatings and show no correlation between the strength of a magnetic field applied parallel to the mirror's surface and BF amplitude. In addition to assisting in the selection of suitable components for the final design, our LHP promises more sensitive results than previous experiments and may very well be the basis for the very first detection of VMB.
\end{abstract}

\section{Introduction}

The classical vacuum is a region of space devoid of matter and any physical fields that carry energy and momentum. This "free space" is therefore the lowest possible energy state of a classical system and acts as the reference state for the permittivity of a material making its relative permittivity identically one. In quantum electrodynamics (QED), a more particular QED vacuum is needed to fit the theory. This vacuum again describes a lowest possible energy state but now it is of the electromagnetic (EM) field when the field is quantized. While the EM field is considered continuous in classical theories, quantum theories require the field to be comprised of discrete packets of energy called quanta, more commonly known as photons. The quantization of this field then gives the QED vacuum a relative permittivity that is not one, as is the case for the classical vacuum. Since the propagation of an EM wave is influenced by this value, the QED vacuum is capable of exhibiting material effects such as birefringence. 
Birefringence primarily concerns two optical properties: the polarization of an EM wave and the index of refraction of a material. Polarization refers to the geometrical orientation of a transverse wave's oscillations since the oscillations are perpendicular to direction of propagation. In an EM wave, both the electric and magnetic fields are perpendicular to one another and transverse, so the polarization is defined by convention to be the direction of the electric field vector. The index of refraction is a dimensionless number that describes the ratio of the speed of light in a vacuum to the speed of light in a medium. Birefringence is the optical property of a material when its index of refraction is polarization-dependent. Vacuum magnetic birefringence, then, is the birefringent quality of the QED vacuum in the presence of strong magnetic fields.

Due to the quantum nature of VMB and previous experimental sensitivity levels, VMB has never before been experimentally detected. We believe that performing a modified experiment to the one originally published by Hall, Ye, and $\mathrm{Ma}^{1}$ using advanced gravitational wave detection technology from LIGO and LISA in the ALPS IIc design will give us the sensitivity necessary to measure VMB. The general ALPS design, depicted in Fig. 1, is for the "Light Shining through a Wall" (LSW) experiment that is meant to search for photon oscillations to and from axions - a particle theorized to exist beyond the Standard Model.

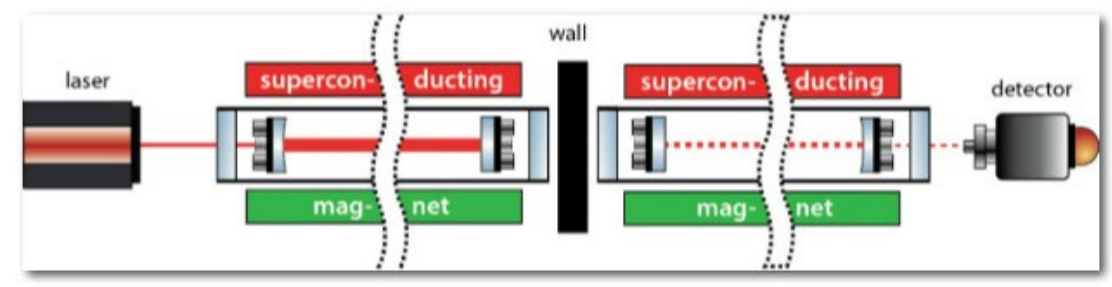

Figure 1. ALPS IIc design for axion detection. ${ }^{2}$ The magnetic field of the production cavity is theorized to produce axions while the regeneration cavity uses the field to generate photons back from axions with a wall separating the two to prevent scattered light from reaching the detector.

A modified version of the ALPS IIc design, shown in Fig. 2, could be used for a VMB experiment. This design has an optical path of approximately $176 \mathrm{~m}$ that will be surrounded by 5.3 T HERA dipole magnets making the expected VMB $\Delta n=1.1 \times 10^{-22}$ and expected path length difference $\Delta l=L \Delta n=2 \times 10^{-20} \mathrm{~m}$. Using infrared lasers with a wavelength of 1064 $\mathrm{nm}$, this translates to a single pass phase difference of $\Delta \varphi=1.16 \times 10^{-13} \mathrm{rad}$.

Detecting such a miniscule effect requires an immensely sensitive experiment that is precisely calibrated. One aspect of this calibration is to account for the BF of external sources that could 
interfere with the final measurement. The illustration in Fig. 2 is simple but it can be seen that the mirrors are one of these sources, so their BF must be known. Our experiment tests the sensitivity

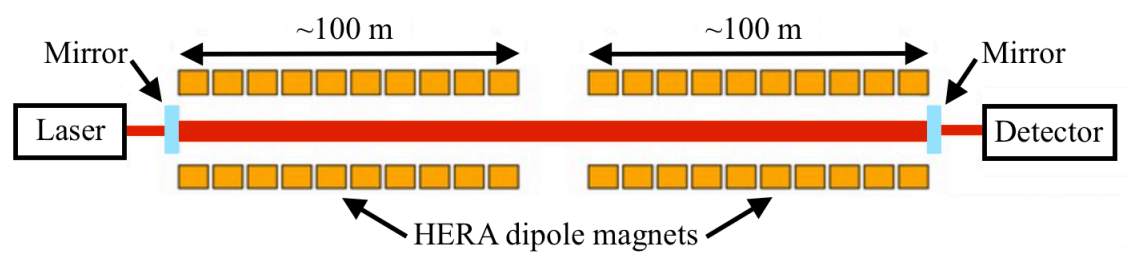

Figure 2. Modified ALPS IIc design for vacuum birefringence detection. ${ }^{3}$ The production and regeneration cavities from the LSW experiment are combined to form a single cavity that would produce the largest single pass phase shift of any VMB experiment.

of a new laser heterodyne polarimeter design that can be used to measure both the birefringence of optical components, like mirrors, and VMB.

The LHP, shown in Fig. 3, does not require a cavity to obtain BF measurements from the BF source, which is labeled as such at the bottom center of the image. Since previous experiments dedicated to detecting BF all required a cavity, this design is more cost effective and provides a method to determine the BF of individual components instead of the two mirrors that comprise a cavity. For VMB detection, a cavity is necessarily present between the mirrors in Fig. 2 to amplify the weak VMB signal. By positioning this cavity to replace the BF source in our LHP, the design is modified for VMB detection.

The rest of the paper discusses the components of the LHP and three primary tests that were conducted to measure the sensitivity of the LHP using a mirror as the BF source. In the first case, we discuss measuring BF variations across the source mirror's surface, and in the second, determining any magnetic effects on the mirror's BF. The final study was performed to further isolate the mirror's BF signal from noise sources.

\section{Apparatus}

Our laser heterodyne polarimeter, as seen in Fig. 3, consists of two primary components: a path for laser locking and a path for signal detection.

\section{Laser Locking}

Heterodyne interferometry uses the offset frequency (beat note) between two independent lasers as a tool for various measurements. While each of our lasers operate at $1064 \mathrm{~nm}$, subpicometer fluctuations create a megahertz beat note. In order to obtain accurate results, this beat 
note must be stabilized so any measurement deviations can confidently be attributed to the source being measured. One method of stabilization is by locking the two lasers, so their offset frequency and phase remain constant.

beam path. Each beam in our apparatus traveled through an identical set of components before being superimposed beginning with a Faraday isolator to prevent backscattered light from damaging the laser. The beams then traveled through a half-wave plate (HWP), which rotated the polarization of the incident light. One field then had p-polarized light, represented by the red line, while the other had s-polarized light, represented by the blue line. The glass plates reduced the power of each laser by one order of magnitude leaving the beams with a power of approximately $10 \mathrm{~mW}$. They were then guided by steering mirrors into the first polarizing beamsplitter (PBS) where they overlapped and propagated coherently to the rest of the phase lock loop (PLL) path and the signal path. In order for the photodetector to detect a beat between the two orthogonally-polarized beams, they must first be projected into the same plane. A HWP set to $22.5^{\circ}$ followed the combining PBS, which rotated the beams by $45^{\circ}$. With the use of another PBS, the s-polarization of each beam was selected giving the PLL PD a linearly polarized field containing equal power contributions from each laser.

phase lock. After the optical signal had been acquired to the PLL PD, several electronic components were used stabilize the beat note with specifications detailed in Table 1. The PD first converted the optical signal to an electrical one that mixed with a signal of the same frequency from the function generator. Our chosen carrier frequency was $5 \mathrm{MHz}$ because it was well within the bandwidth of all the components. The mixer then output the sum and difference frequencies of the two inputs before the low pass filter (LPF) selected the difference frequency, $0 \mathrm{~Hz}$, with minimal loss.

This signal was then fed into an analog PLL made in-house that returned two outputs to minimize the drift of the beat between the two lasers. One channel output a "slow" correction signal to the temperature control of the laser in Fig. 4 (Laser 2 in Fig. 3). This signal was used to offset the slight drift in room temperature over hours. The second channel output a "fast" correction signal that quickly adjusted the voltage across the piezo crystal in the laser to offset random phase fluctuations. With these controls in place, the beat between the two lasers remained in phase with the local oscillator. From the stability of the function generator, we know the frequency of the beat was also locked. 

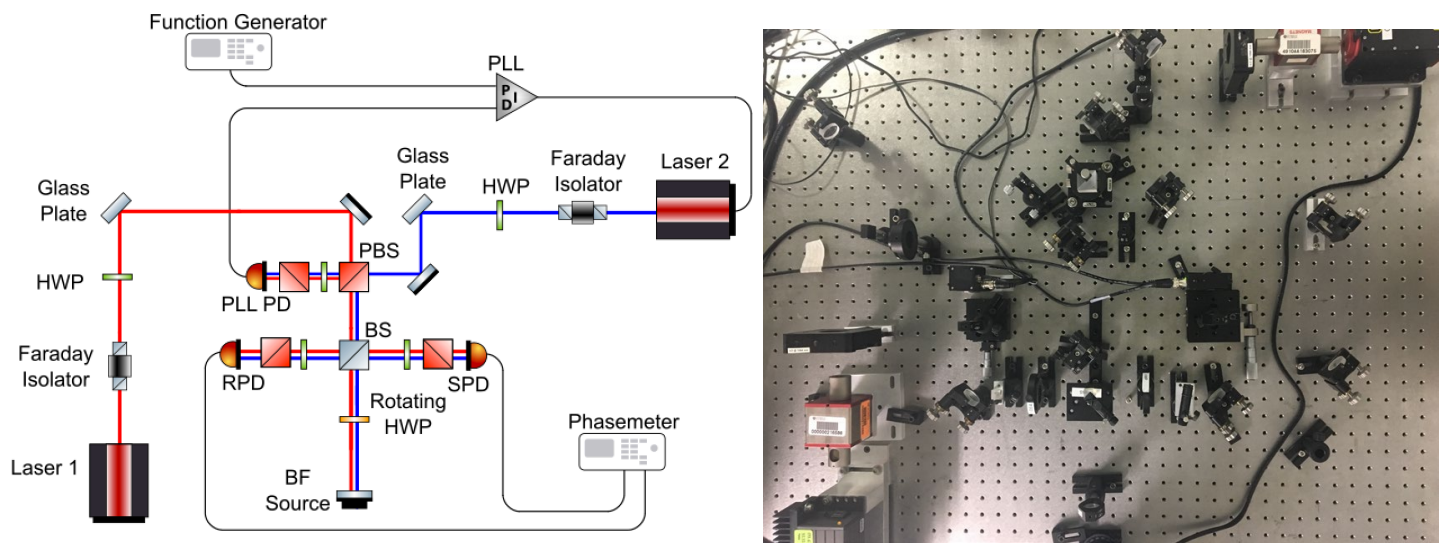

Figure 3. Left: LHP schematic used to measure mirror BF (BF source). From their emission, the lasers are prepared to be polarized, which occurs at the first PBS. They then travel coherently on two paths: one to the PLL PD for laser locking, and another to the BS for signal detection where they are split once more.

Right: View of our LHP experimental apparatus from above. The PLL PD is out of range but is located above the upper left corner.

Table 1. Laser locking electrical component specifications

\begin{tabular}{|c|c|c|c|}
\hline Component & Data Specifications & & \\
\hline \multirow{3}{*}{ Mini-Circuits ZAD-6+ Mixer } & $f_{L O} / f_{R F}(M H z)$ & $f_{I F}(M H z)$ & LO Power \\
\hline & $0.003-100$ & $\mathrm{DC}-100$ & $+7 \mathrm{dBm}$ \\
\hline & Passband & - & Impedance \\
\hline \multirow[t]{2}{*}{ Mini-Circuits BLP-5+ LPF } & $\begin{array}{c}\mathrm{DC}- \\
22 \mathrm{MHz}\end{array}$ & - & $50 \Omega$ \\
\hline & Frequency Range & Amplitude Range (Vpp) & Impedance \\
\hline \multirow[t]{2}{*}{$\begin{array}{l}\text { SRS DS345 Synthesized } \\
\text { Function Generator }\end{array}$} & $\begin{array}{c}1 \mu \mathrm{Hz}- \\
30.2 \mathrm{MHz}\end{array}$ & $\begin{array}{l}0.01-10 \\
(@ 50 \Omega)\end{array}$ & $50 \Omega / 1 \mathrm{M} \Omega$ \\
\hline & Bandwidth & Gain & $N E P$ \\
\hline EOT-3000A Amplified PD & $\begin{array}{l}30 \mathrm{kHz}- \\
1.5 \mathrm{GHz}\end{array}$ & $\begin{array}{c}770 \mathrm{~V} / \mathrm{W} \\
(@ 1064 \mathrm{~nm})\end{array}$ & $\sim 39 \mathrm{nW} / \sqrt{ } \mathrm{Hz}$ \\
\hline
\end{tabular}




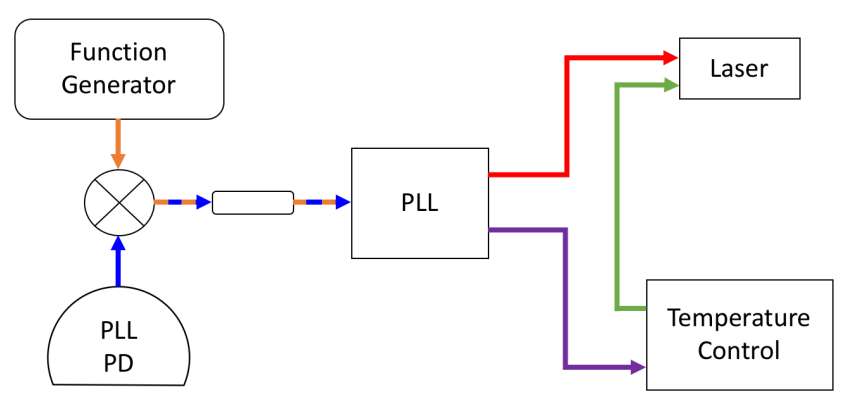

Figure 4. Laser locking electronics schematic. Signals from the PLL PD and function generator are combined in a mixer. A low pass filter selects the difference frequency to input into the PLL. One feedback loop connects directly to the piezo crystal in the laser while the other adjusts the laser's temperature control.

\section{Signal Detection}

beam path. Once the two beams combined at the first PBS, one path was used for locking while the other path was for signal detection. The subsequent procedure follows that of Hollis et. al. ${ }^{4}$ The two orthogonally-polarized, phase-locked beams at this stage, shown in Fig. 3, can be represented through the Jones matrices as

$$
\tilde{\mathbf{E}}=\left(\begin{array}{l}
1 \\
0
\end{array}\right) A_{p} e^{i \omega t}+\left(\begin{array}{l}
0 \\
1
\end{array}\right) A_{s} e^{i(\omega+\Omega) t}
$$

where $A_{p}$ and $A_{s}$ are real amplitudes and $\Omega$ is the carrier frequency. The reflected field from a beam splitter (BS) that further divides the experiment into reference and signal paths is then

$$
\tilde{\mathbf{E}}_{\mathrm{R}}=\frac{1}{\sqrt{2}}\left(\left(\begin{array}{l}
1 \\
0
\end{array}\right) A_{p}+\left(\begin{array}{l}
0 \\
1
\end{array}\right) A_{s} e^{i \Omega t}\right) e^{i \omega t}
$$

The reference path simply projected the field into a single plane, as with the locking path, so a PBS selected the s-polarization after the field traveled through a HWP rotated at $22.5^{\circ}$. The field on the reference detector, RPD, after this combination of components then gives an intensity of

$$
I_{\mathrm{RPD}}=\left|\tilde{\mathbf{E}}_{\mathrm{RPD}}\right|^{2}=\frac{1}{4}\left(A_{p}^{2}+A_{s}^{2}\right)-\frac{1}{2} A_{p} A_{s} \cos \Omega t
$$

The RPD signal was used as a standard measurement for noise inherent to the setup. Since we only desired to detect the BF from the source mirror, a BB1-E03 Broadband Dielectric mirror 
from Thorlabs, any extraneous effects could be eliminated by subtracting the phase information on RPD.

The signal path began with the BS transmission field:

$$
\tilde{\mathbf{E}}_{\mathrm{T}}=\frac{i}{\sqrt{2}}\left(\left(\begin{array}{l}
1 \\
0
\end{array}\right) A_{p}+\left(\begin{array}{l}
0 \\
1
\end{array}\right) A_{s} e^{i \Omega t}\right) e^{i \omega t}
$$

The field propagated through another HWP but now one rotated at an angle $\theta_{\pi}$ before reflecting off the source mirror rotated at $\theta_{m}$. The phase of each polarization after this reflection is denoted $\phi_{x}$ and $\phi_{y}$. The field, after propagating back through the rotated HWP, reflects at the BS and enters an equivalent HWP-PBS combination as before all PDs. This field, represented by

$$
\begin{gathered}
\tilde{\mathbf{E}}_{\mathrm{SPD}}=\frac{i}{4 \sqrt{2}}\left\{\left(e^{i \phi_{x}}-e^{i \phi_{y}}\right)\left(A_{p}+A_{s} e^{i \Omega t}\right) \cos 2 \gamma-\left(e^{i \phi_{x}}-e^{i \phi_{y}}\right)\left(A_{p}-A_{s} e^{i \Omega t}\right) \sin 2 \gamma\right. \\
\left.+\left(e^{i \phi_{x}}+e^{i \phi_{y}}\right)\left(A_{p}-A_{s} e^{i \Omega t}\right)\right\} e^{i \omega t}
\end{gathered}
$$

where $\gamma=2 \theta_{\pi}-\theta_{m}$, now contains a phase-shifted beat note compared to the RPD signal because of the mirror's BF. In the case when this BF, $\delta \equiv \phi_{x}-\phi_{y}$, is small, we can approximate the intensity on the signal PD to be

$$
\begin{aligned}
I_{\mathrm{SPD}} \approx & \frac{1}{8}\left(A_{p}^{2}+A_{s}^{2}\right)-\frac{1}{4} A_{p} A_{s}(\delta \cos 2 \gamma \sin \Omega t+\cos \Omega t) \\
& \approx \frac{1}{8}\left(A_{p}^{2}+A_{s}^{2}\right)-\frac{1}{4} A_{p} A_{s} \cos (\Omega t+\phi)
\end{aligned}
$$

where $\phi=2 \delta \cos 2 \gamma=2 \delta \cos \left(4 \theta_{\pi}-2 \theta_{m}\right)$.

By rotating the HWP at a constant angular velocity such that $\theta_{\pi}=\omega_{\pi} t$, we show that

$$
\phi=\delta \cos \left(4 \omega_{\pi} t-2 \theta_{m}\right)
$$

Thus, with ideal components, the mirror's BF produced a signal at four times the rotation rate of the HWP with amplitude $\delta$. We were able accomplish this rotation using a set of Thorlabs components: a DDR05 rotation mount, a KBD101 K-Cube Brushless DC Servo controller, and an APT system software.

With imperfect components, Eq. 10 deviates slightly. An error in the rotating HWP can be modeled by adjusting its retardation to $\pi+\epsilon$. Taking only first order terms in $\delta$ and $\epsilon$, an extra 
factor of arises in the SPD intensity when again considering $\delta$ to be small. This modifies Eq. 10 to be

$$
\phi=\delta \cos \left(4 \omega_{\pi} t-2 \theta_{m}\right)-2 \epsilon \cos 2 \omega_{\pi} t
$$

showing a signal from the HWP error appear at twice its rotation rate with an amplitude of $2 \epsilon$.

In order to ensure the mirror's BF signal does not appear at a frequency laced with other spurious signals, we extended the SPD intensity to include the next higher order term in $\epsilon$. This led to a beat note phase shift of

$$
\phi \approx \delta \cos \left(4 \omega_{\pi} t-2 \theta_{m}\right)-2 \epsilon \cos 2 \omega_{\pi} t-\frac{\epsilon^{3}}{3} \cos 6 \omega_{\pi} t
$$

which identifies an additional peak at six times the HWP rotation due to its retardation error, but no additional signal at $4 \omega_{\pi} t-$ the frequency of the mirror BF signal.

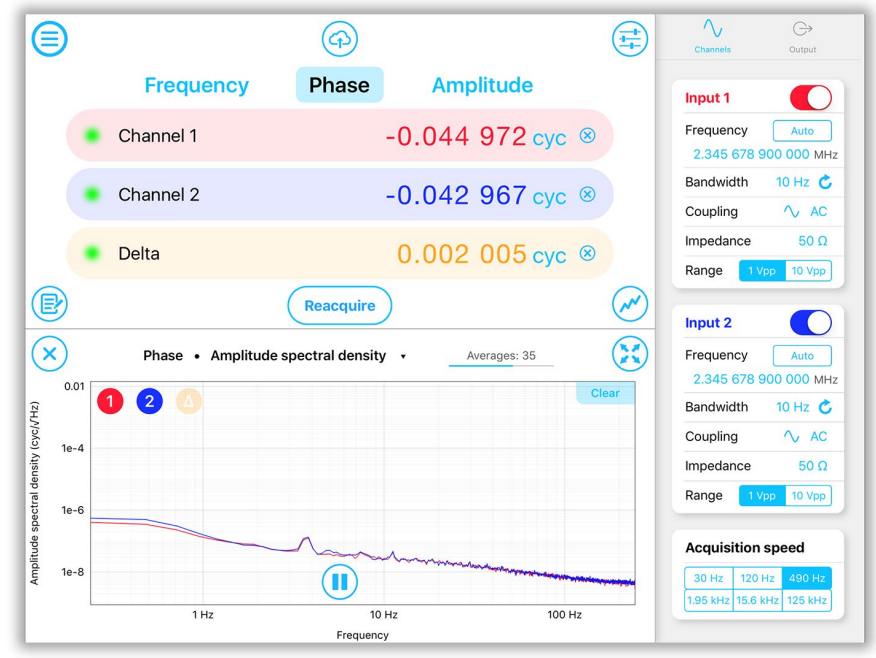

Figure 5. Moku:Lab Phasemeter iPad interface. ${ }^{5}$ Input 1 and 2 had the same settings: $5 \mathrm{MHz}$ reference frequency, $10 \mathrm{kHz}$ bandwidth, AC coupling, $50 \Omega$ impedance, and a range of $1 \mathrm{Vpp}$.

phase processing. To gather phase information from the RPD and SPD, we used a Moku:Lab Phasemeter from Liquid Instruments. This instrument tracked the phase, frequency, and amplitude of each PD through two independent channels. The interface was provided through an iPad application, seen in Fig. 5, and provided optimal control of various parameters.

Each channel locked onto a reference frequency equal to that of our beat note with a 1 $\mathrm{Vpp}$ range since the maximum RPD and SPD voltages were approximately $0.6 \mathrm{~V}$ and $0.3 \mathrm{~V}$ respectively. The sampling rate was set to the 120 samples per second (S/s) setting although the 
true value was closer to $122.07 \mathrm{~S} / \mathrm{s}$. Data was saved as a CSV file which was then used for analysis in MATLAB. To convert the raw phase data into plots containing the amplitudes of various BF signals, we took the discrete Fourier transform (DFT) of the phase difference between the two channels.

\section{Spatial Variance of the Mirror's BF}

One test performed on the mirror involved determining how its BF varied when probed at different locations on its surface.

\section{Methods}

In order to obtain a BF surface map, we needed to be able to adjust the mirror's position in the 2D plane perpendicular to the incident light. We used a combination of a Thorlabs 6XS mirror mount and a Newport 460-XYZ mount to accomplish this with enough translational freedom to cover a significant portion of the mirror's surface. We then constructed a $4 \times 4$ grid of points, shown in Fig. 6, to effectively probe $144 \mathrm{~mm}^{2}$, which maximized our area covered while maintaining that the beam was fully reflected. A two-minute measurement was taken at each point.

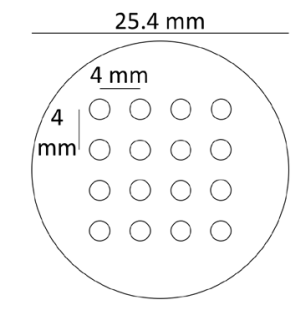

Figure 6. Actual-size image of mirror with $4 \times 4$ spatial grid of beam locations. To ensure the beam was not clipped, we approximated it to be contained within a circle of radius $1 \mathrm{~mm}$ at the mirror's surface.

\section{Results}

It is shown from Fig. 7 that the birefringence across the mirror's surface varied approximately 2 mrad from 22.4 to $24.4 \mathrm{mrad}$. In comparison, repeated measurements on a single point showed a standard deviation of approximately $0.2 \mathrm{mrad}$. Both plots showed the maximum BF at the point diagonal from the bottom and left while the minimum BF was at the point directly above it. 

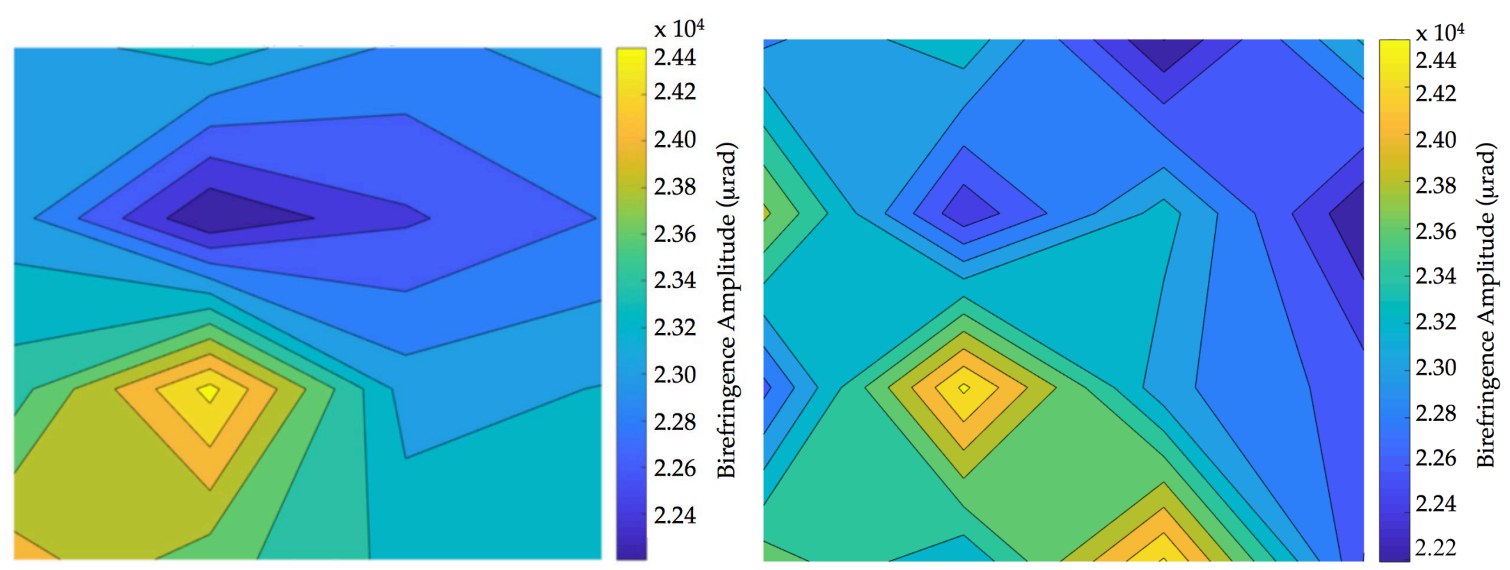

Figure 7. Interpolated plots of spatial BF variance. Over multiple runs, the mirror's total BF variance and average $\mathrm{BF}$ remained constant at $2 \mathrm{mrad}$ and $23 \mathrm{mrad}$ respectively. The gradients were created through MATLAB.

\section{Conclusions}

These values were consistent over multiple runs covering all 16 points before measuring another point again. The surface fluctuations could therefore be attributed to imperfections over random error. Since this mirror, a BB1-E03 Broadband Dielectric mirror from Thorlabs, was composed of a polished glass substrate under alternating layers of reflective coatings, we concluded the imperfections were due to errors in the coating layers.

\section{Magnetic Effect on the Mirror's BF}

The $5 \mathrm{~T}$ magnets present in the modified ALPS IIc design from Fig. 2 will produce strong fields outside of their intended target area. The mirrors will therefore be subject to some amount of these stray fields. In order to precisely characterize all BF effects and eliminate the possibility of a false positive when attempting to detect VMB, we examined how a mirror's BF varied when placed in a magnetic field parallel to its surface.

\section{Apparatus and Methods}

First, we wanted to ensure all measurements contained contributions strictly from the magnetic effect, so we inserted a telescope between the rotating HWP and source mirror. This expanded the beam so its cross-sectional area at the mirror was nearly as large as the mirror's surface itself. With this beam expander in place, the minor surface variations were averaged out giving us a more stable measurement for the mirror's BF on its own. 
To replicate the stray fields present on the mirror's surface, we obtained strong magnets and created a device to hold them in place over a range of distances. The four magnets we used were 2" x 1" x 3/8” N52-Neodynium magnets (BY0X06-N52) from K\&J Magnets, Inc. that each produced a surface field strength near the 3400 Gauss advertised. We were able to measure this with a Gauss meter and calculate it using the following equation provided by K\&J Magnets, Inc.:

$$
B=\frac{B_{r}}{\pi} \tan ^{-1}\left(\frac{L W}{2 z \sqrt{4 z^{2}+L^{2}+W^{2}}}\right)-\tan ^{-1}\left(\frac{L W}{2(D+z)^{2} \sqrt{4(D+z)^{2}+L^{2}+W^{2}}}\right) .
$$

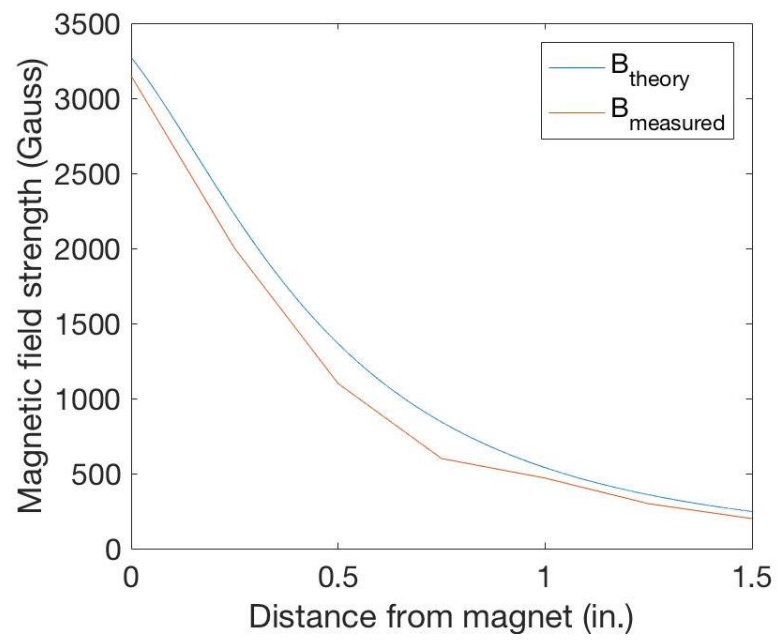

Figure 8. Plot of magnetic field strength vs distance from a magnet pole for a single N52-neodymium magnet. Neither the measured nor the theoretical calculation reach 3400 Gauss at the magnet's surface, but the two curves align nonetheless.

In Eq. 13, $B_{r}$ is the remanence field; $L, W$, and $D$ are the length, width, and thickness of the magnet respectively; and $z$ is the distance away from a pole assuming the poles are oriented with the axis of the thickness. The units of $L, W, D$, and $z$ are arbitrary so long as they are all the same. The theoretical and measured fields for a single magnet are plotted in Fig. 8.

The holders, shown in Fig. 9, had spaces to fit each magnet independently, which would produce a combined field that is parallel to the surface of the mirror. The holders also had holes for two poles and side screws that allowed us to adjust the distance of the magnets from the mirror in eighth-inch increments using distance markings on the poles. In addition, the casings were made out of phenolic making neither them nor the mirror mount magnetic. 


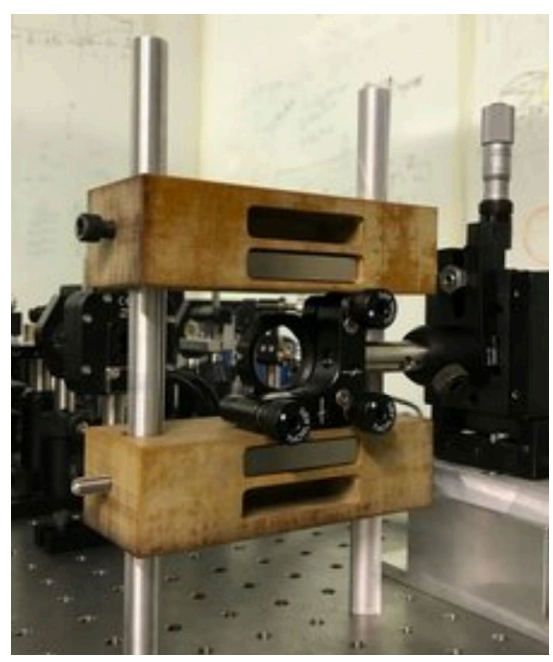

Figure 9. Lab photograph of magnet holder surrounding the mirror. Rulers at the poles allowed us to vary the distance in eighth-inch increments. The casings themselves were crafted out of a non-magnetic material named phenolic.

With all four magnets in place, we took two-minute measurements at eighth-inch increments. The nearest position is depicted in Fig. 9 and is when the closest magnet is one inch from the center of the mirror. The furthest position is when the bottom casing is sitting on the table, which makes the closest magnet 2.5 inches from the mirror's center.

\section{Results}

As seen in Fig. 10, the BF of a New Focus 5104 mirror remains essentially unchanged over a range of nearly 1000 Gauss. The uncertainty in field strength arises from the sixteenth-inch uncertainty in our distance measurements while the uncertainty in our BF amplitude was determined after repeated measurements. The mean BF amplitude of 18,200 $\pm 400 \mu$ rad was near both the initial and final values even over magnetic field strengths spanning greater than $1 \mathrm{kG}$.

\section{Conclusions}

The New Focus 5104 mirror used for this experiment was another commercially accessible mirror similar to the BB1-E03 in that both were highly reflective for $1064 \mathrm{~nm}$ light due to their composition of reflective coatings above a glass substrate. The stability of the measurements shown in Fig. 10 allowed us to conclude that for magnetic field strengths up to $1.2 \mathrm{kG}$, both the substrate and reflective coatings of a New Focus 5104 mirror were unaffected by field strength. 


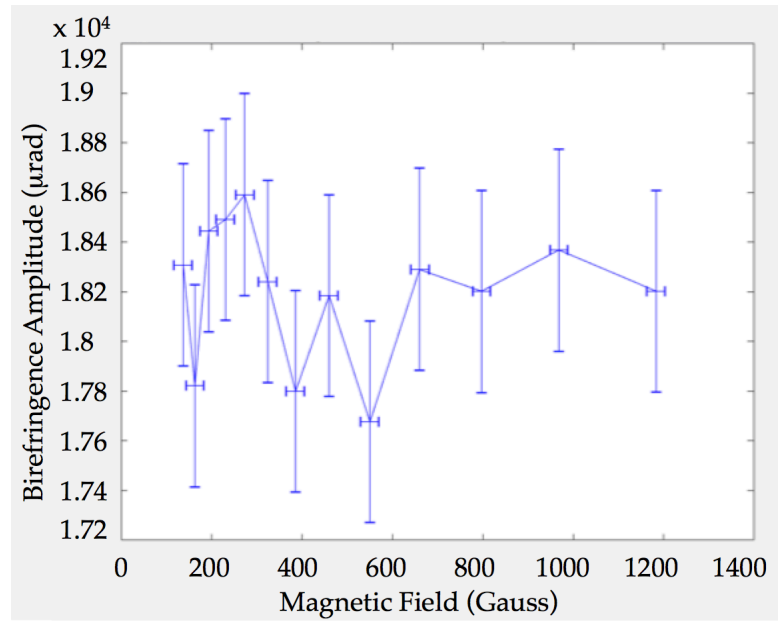

Figure 10. Plot of BF vs magnetic field strength for the New Focus 5104 mirror. No correlation between BF and field strength can be determined with our error in this range of field strengths.

\section{Isolation of the Mirror's BF Signal}

From Eq. 11, we expect BF signals at twice and four times the rotation rate of the HWP and from Eq. 12, we can even expect a small signal at six times this rate. When observing the DFT of the phase difference between our two channels, however, we spot these peaks along with others at integer multiples of the rotation rate, as seen in Fig. 11.

\section{Methods}

In an attempt to confirm the amplitude of our mirror's birefringence only, we utilized the notion that under constant rotation, we can set $\theta_{m}=\omega_{m} t$ so Eq. 11 becomes

$$
\phi=\delta \cos \left(4 \omega_{\pi} t-2 \omega_{m} t\right)-2 \epsilon \cos 2 \omega_{\pi} t
$$

meaning the mirror's BF signal can be moved off $20 \mathrm{~Hz}$. We used a function generator connected to a stepper motor that controlled the gears on a mirror mount to set the mirror's rotation rate to $3.5 \mathrm{~Hz}$ in the opposite direction of the HWP rotation.

\section{Results}

In accordance with Eq. 14, the mirror's birefringence moved to $27 \mathrm{~Hz}$ with an amplitude of $\delta$, 


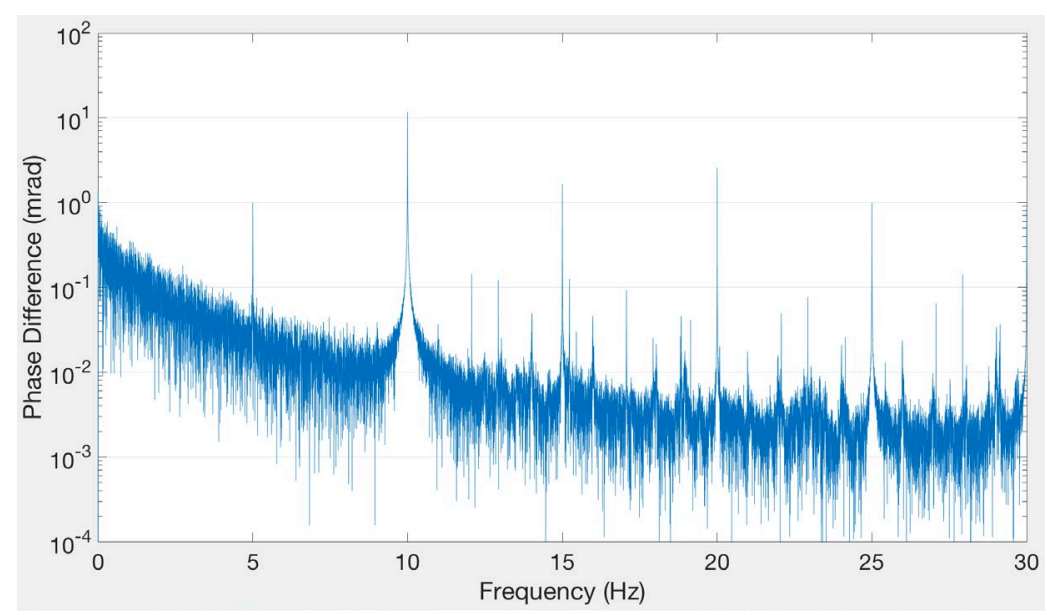

Figure 11. Phase difference DFT showing strong peaks at 10 and $20 \mathrm{~Hz}$ from the HWP error and mirror respectively. The origin of other significant peaks at higher harmonics of $5 \mathrm{~Hz}$ are being investigated.

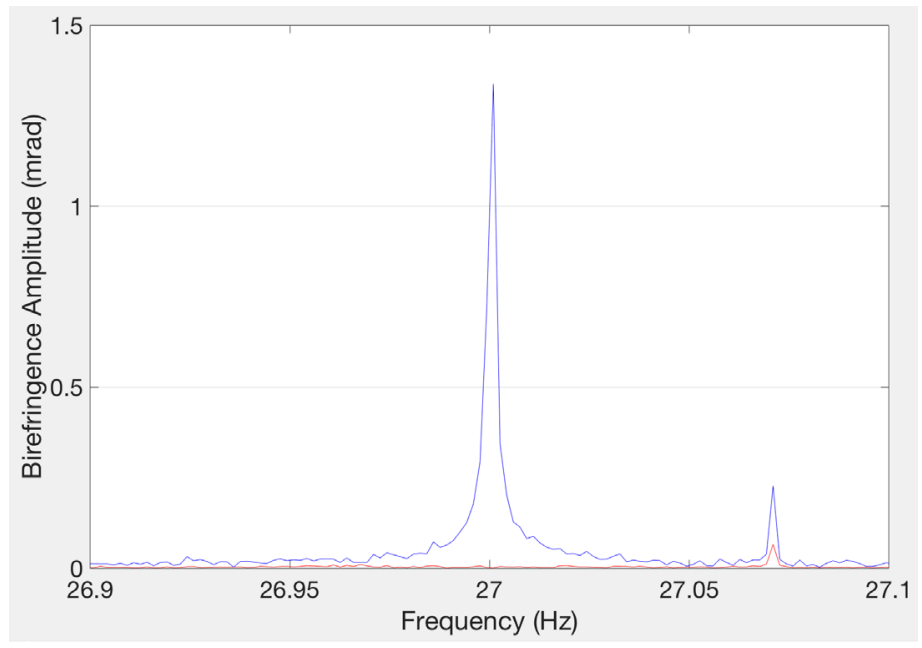

Figure 12. Isolated mirror BF signal. The red line (below) represents the signal BF when only the HWP is rotating (5 $\mathrm{Hz}$ ) while the blue line (above) represents the signal BF when both the HWP and mirror are rotating $(5 \mathrm{~Hz}, 3.5 \mathrm{~Hz})$.

as seen in Fig. 12. The BB1-E03 mirror's BF was determined to be $1.34 \mathrm{mrad}$ with phase noise of $2 \mu \mathrm{rad} / \sqrt{\mathrm{Hz}}$. From this, we can reach a sensitivity of $0.1 \mu \mathrm{rad}$ in $400 \mathrm{~s}$ averaging time, matching the precision of previous experiments ${ }^{6}$ without the complications of a vacuum cavity.

Unfortunately, including the mirror's own rotation rate introduced peaks at its fundamental frequency and higher harmonics, as seen in Fig. 13. We know that a signal will appear at the mirror's rotation rate if the rotation axis is not perfectly aligned with the incident beam but are unclear about the origin of the other peaks. 


\section{Conclusions}

By rotating the mirror at a fixed rate, we successfully shifted the mirror's BF signal to a frequency isolated from any HWP harmonics. From this, we concluded that our LHP design one that does not require a cavity - can directly measure mirror BF with a sensitivity comparable to that of previous experiments.

\section{Outlook}

While we were able to successfully measure the mirror's birefringence at an independent frequency with no other BF contributions, we would like to eliminate the excess noise and higher harmonics to create a more sensitive experiment for VMB detection. The magnets in ALPS IIc will have the capability of fluctuating between $50 \%$ and $100 \%$ power at a slow rate $(\sim 1 \mathrm{mHz})$, which would produce sidebands around the HWP rotation frequency. The sidebands would also be at a frequency uncorrupted by excess rotation noise. We can simulate this procedure by keeping the mirror at a fixed angle and inputting an electro-optic amplitude modulator (EOAM) after the rotating HWP and before the source mirror. Early findings show promise that even a weak signal from sending a $0.01 \mathrm{Vpp}$ sine wave at $1 \mathrm{~Hz}$ to the EOAM can be distinguished with a BF of $400 \mu \mathrm{rad}$.

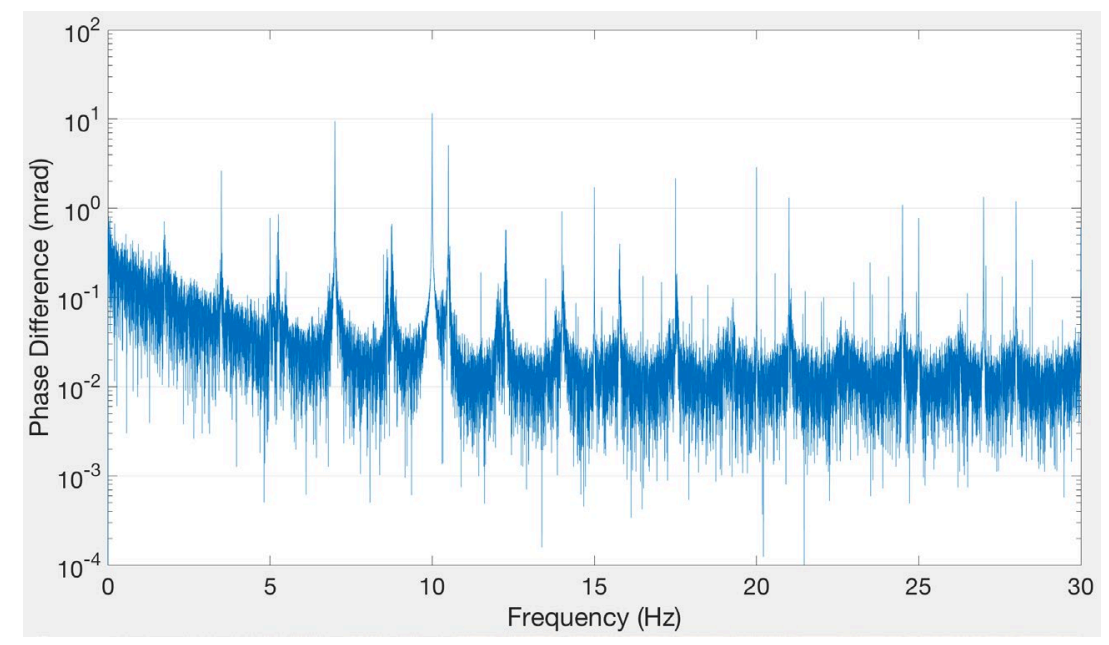

Figure 13. DFT spectrum with both the mirror rotating at $3.5 \mathrm{~Hz}$ and the HWP rotating at $5 \mathrm{~Hz}$. The $10 \mathrm{~Hz}$ and 20 $\mathrm{Hz}$ amplitudes are nearly equal to what they were in Fig. 11 but are surrounded by more noise from the combined harmonics of the mirror's and HWP's rotation. 


\section{Acknowledgements}

I would first like to thank Guido Mueller for giving me the opportunity to work on this project two years ago. I would also like to thank Hal Hollis for helping me understand the fundamentals of this experiment, Harrison LaBollita for his contributions to the experiment over last summer, and Claire Baum for constructing the foundations of the experiment two summers ago. Finally, I would like to thank my family for their love and support, and my girlfriend, Hannah, for encouraging me through the entire writing process and beyond.

This material is based upon work supported by the Heising-Simons Foundation under Grant No. 0009872 .

\section{References}

${ }^{1}$ J. L. Hall, J. Ye, and L.-S. Ma, Phys. Rev. A 62, 013815 (2000)

${ }^{2}$ ALPS, Present and Future of ALPS II, https://alps.desy.de/e191931/

${ }^{3}$ G. Mueller et al., APS April Meeting 2016, 61, Available online at: http://meetings.aps.org/link/BAPS.2016.APR.U16.4 (2016)

${ }^{4}$ H. Hollis, G. Alberts, D. Tanner, G. Mueller, arXiv:1710.03801v1 [physics.ins-det] (2017)

${ }^{5}$ Moku:Phasemeter, http://www.liquidinstruments.com/phasemeter/

${ }^{6}$ A. J. Fleisher, D. A. Long, Q. Liu, and J. T. Hodges, Phys. Rev. A 93, 1-7 (2016) 